\title{
HIV-Related Neurological Syndromes Reduce Health-Related Quality of Life
}

\author{
Rupang Pandya, Hartmut B. Krentz, M. John Gill, Christopher Power
}

\begin{abstract}
Background: Human immunodeficiency virus (HIV) infection frequently results in neurological complications but the impact of different neurological syndromes on patients' quality of life remains unknown. Methods: We investigated health-related quality of life (HRQoL) parameters among HIV/Acquired Immune Deficiency Syndrome (AIDS) patients with and without neurological disease, including 11 dimensions of HRQoL within the Medical Outcomes Short-form Health Survey-HIV. Results: Comparisons of sociodemographic and systemic clinical variables did not differ between HIV/AIDS patients with $(n=94)$ and without $(n=75)$ neurological disease. However, patients with neurological diseases exhibited significantly lower HRQoL scores compared to matched controls, which was most evident among HIV/AIDS patients with cognitive impairment and sensory neuropathy. Prospective analysis revealed diminishing HRQoL scores prior to neurological diagnosis followed by a progressive and sustained improvement in HRQoL scores after intervention over a 96-week period. Conclusions: These studies indicate that while HIV-related neurological diseases are associated with reduced HRQoL scores, enhanced neurological care has a positive impact on HIV/AIDS patients' overall well-being.
\end{abstract}

\begin{abstract}
RÉSUMÉ: Les syndromes neurologiques propres à l'infection par le VIH diminuent la qualité de vie reliée à la santé. Introduction: L'infection par le virus de l'immunodéficience humaine (VIH) comporte des complications neurologiques et l'impact de différents syndromes neurologiques sur la qualité de vie des patients atteints demeure inconnu. Méthodes: Nous avons évalué des paramètres de la qualité de vie reliés à la santé (QVRS) chez des patients porteurs du VIH/SIDA, avec et sans maladie neurologique, dont 11 mesures de la QVRS du Medical Outcomes Short-form Health Survey-HIV. Résultats: Il n'existait pas de différence entre les patients atteints de VIH/SIDA avec $(\mathrm{n}=94)$ ou sans $(\mathrm{n}=75)$ maladie neurologique quant aux variables sociodémographiques et cliniques systémiques. Cependant les patients atteints de maladies neurologiques avaient des scores significativement plus faibles pour la QVRS par rapport à des témoins appariés. Cette différence était plus marquée chez les patients atteints de VIH/SIDA qui présentaient une atteinte cognitive et une neuropathie sensitive. Une analyse prospective a montré des scores de la QVRS plus faibles avant le diagnostic neurologique et une amélioration progressive soutenue des scores après intervention sur une période de 96 semaines. Conclusions: Bien que les maladies neurologiques reliées au VIH soient associées à des scores plus bas à l'évaluation de la QVRS, ces études indiquent que des soins neurologiques adéquats ont un impact positif sur le bien-être général des patients atteints de VIH/SIDA.
\end{abstract}

Can. J. Neurol. Sci. 2005; 32: 201-204

Despite the widespread use of highly active antiretroviral therapy (HAART) among patients with human immunodeficiency virus (HIV) infection, neurological disorders remain important and disabling aspects of HIV-related disease. ${ }^{1}$ Indeed, the prevalence of HIV-related neurological syndromes including sensory neuropathy and cognitive impairment, have increased in recent years as HIV/Acquired Immune Deficiency Syndrome (AIDS) patients live longer. ${ }^{2}$ Subjective measures of health and well-being including health quality of life (HRQoL) parameters have become important in assessing health status during systemic HIV infection, showing that HIV infection is accompanied by lower HRQoL ratings, especially as immunosuppression progresses. ${ }^{3}$ However, the influence of neurological disorders on HRQoL among HIV/AIDS patients has not been studied despite the substantial effects of

From the Departments of Psychiatry (RP), Anthropology (HBK), Medicine (MJG) and Clinical Neurosciences (CP), University of Calgary, Calgary, AB, Canada. ReCEIVED JunE 11, 2004. ACCEPTED IN FINAL FORM NOVEMBER 8, 2004. Reprint requests to: C. Power, Department of Clinical Neurosciences, HMRB 150, 3330 Hospital Dr. NW, University of Calgary, Calgary, Alberta, Canada T2N 4N1 
neurological complications on disease course. Nonetheless, neuropsychological impairment has been associated with lower HRQoL scores in some studies. ${ }^{4,5}$ The objective of our study was to examine the impact of different neurological syndromes on HRQoL by studying HIV/AIDS patients with and without neurological disease that were matched in terms of level of immunosuppression and treatment regimens.

\section{METHODS}

The Southern Alberta Clinic is a comprehensive and centralized community outpatient HIV clinic that provides care to all HIV seropositive patients in southern Alberta, Canada with clinical assessments at regular intervals (three to four months) including antiretroviral and related medications as well as laboratory investigations (CD4/CD8 lymphocyte counts, viral loads, etc.) without patient financial cost. The present study was nested in a prospective parent study that examined the socioeconomic impact of HIV/AIDS on adults ( $>18 \mathrm{yr}$.). ${ }^{6}$ Inclusion in the parent study was voluntary and independent of health status including neurological disease. Consent for participation was reviewed at the outset, which was approved by the University of Calgary Ethics Committee. Sociodemographic, clinical, and HRQoL data were collected at the initial visit and at subsequent visits at approximate 16-week intervals between 1999 and 2002. Patient HRQoL was evaluated using the Medical Outcomes Short-form Health Survey-HIV (CAlbert W. Wu, Johns Hopkins University, Version 2.97), an instrument that is a 35-item questionnaire containing 11 subscales scored independently. In situations where the patient had difficulty with the instrument, the interviewer and/or caregiver assisted the patient. Higher scores on each dimension (range: 0 to 100) indicate higher levels of functioning.

Patients were selected on the basis of the presence or absence of a concurrent neurological diagnosis made by a clinical neurologist based on established criteria. $^{7}$ The Neurological Disease Group (NDG) was subdivided into the following categories: (a) HIV-related cognitive impairment including HIVassociated dementia or minor cognitive motor disorder; (b) HIVsensory neuropathy including distal sensory polyneuropathy or antiretroviral toxic neuropathy and (c) Miscellaneous NDG including seizures, migraine, mononeuritides, transient ischemic attack/stroke, tremor, which developed after the diagnosis of HIV infection. Patients who demonstrated severe cognitive impairment (Memorial Sloan Kettering dementia severity score $\geq 2)^{8}$ were excluded from this analysis.

Neurological disease-free controls were compared to the NDG, HIV-related cognitive impairment, and HIV-sensory neuropathy groups. If multiple neurological diagnoses were assigned at different times, the date of the first neurological diagnosis was used. A subset of patients in the parent study, without neurological disease, were selected on the basis of age (>35 years), sex, education, CD4 count $\left(<500 / \mathrm{mm}^{3}\right)$, and HIV disease duration ( $>36$ months) as a matched control group (Controls). Statistical analyses using parametric and nonparametric tests were performed using Graph-Pad Instat version 3.0 (Graph Pad software, San Diego, CA). $p$ values greater than 0.05 were considered nonsignificant.

\section{RESULTS}

Given that neurological disease is associated with increasing immunosuppression and age among HIV/AIDS patients and HRQoL scores decline with progressive immunosuppression, it was essential to ensure that the Control and NDG patients were similar in terms of risk factors for HIV infection, level of immunosuppression, and antiretroviral treatment. Selected Controls did not differ significantly from the NDG with respect to gender, age, HIV risk factor, ethnicity, educational level, mean CD4 nadir, duration of HIV infection and the number of patients receiving HAART (Table). Thus, sociodemographic and clinical features of systemic disease did not differ between NDG and Control groups. Similarly, stratified NDG groups including HIVsensory neuropathy $(n=41)$ and cognitive impairment $(n=19)$ also did not differ in age, CD4 count, education, nor duration of infection from the Control group (data not shown). Comparisons of HRQoL dimension scores among Controls and the NDG revealed that patients with neurological syndromes exhibited significantly lower HRQoL scores in ten of 11 dimensions compared to Controls (Figure) at the onset of the study. Moreover, the HIV-sensory neuropathy group showed lower HRQoL scores compared to the Control group in all dimensions

\section{Table: Sociodemographic and clinical features of neurological disease-free control and neurological disease HIV/AIDS groups}

\begin{tabular}{|c|c|c|c|}
\hline & $\begin{array}{l}\text { CONTROL } \\
(\mathrm{N}=75)\end{array}$ & $\begin{array}{l}\text { NEUROLOGICAL } \\
\text { DISEASE }(\mathrm{N}=94)\end{array}$ & P VALUE \\
\hline \multicolumn{4}{|l|}{ Gender } \\
\hline Male & $70(90 \%)$ & $86(91 \%)$ & NS \\
\hline Female & $5(10 \%)$ & $8(9 \%)$ & \\
\hline Age (yr) & $44.6 \pm 6.7$ & $44.6 \pm 9.6$ & NS \\
\hline \multicolumn{4}{|l|}{ Risk Factor } \\
\hline MSM $^{1}$ & $58(77 \%)$ & $72(77 \%)$ & NS \\
\hline Heterosexual & $7(9 \%)$ & $12(13 \%)$ & \\
\hline $\mathrm{IDU}^{2}$ & $8(11 \%)$ & $6(6 \%)$ & \\
\hline Other & $2(3 \%)$ & $4(4 \%)$ & \\
\hline \multicolumn{4}{|l|}{ Ethnicity } \\
\hline Caucasian & $65(86 \%)$ & $89(95 \%)$ & NS \\
\hline Aboriginal & $4(5 \%)$ & $3(3 \%)$ & \\
\hline African-Canadian & $4(5 \%)$ & $0(0 \%)$ & \\
\hline Other & $3(5 \%)$ & $2(2 \%)$ & \\
\hline \multicolumn{4}{|l|}{ Education } \\
\hline$<12$ years & $3(4 \%)$ & $23(25 \%)$ & NS \\
\hline 12 years & $21(28 \%)$ & $20(21 \%)$ & \\
\hline$>12$ years & $50(67 \%)$ & $51(54 \%)$ & \\
\hline Not reported & $1(1 \%)$ & $1(1 \%)$ & \\
\hline CD4 (cells/ $/ \mu \mathrm{l})$ & $308 \pm 179.9$ & $361 \pm 261.6$ & NS \\
\hline HIV Duration (mo.) & $102.4 \pm 43.7$ & $88 \pm 52.8$ & NS \\
\hline \multicolumn{4}{|l|}{ Patients receiving } \\
\hline HAART & $60(80 \%)$ & $71(76 \%)$ & NS \\
\hline
\end{tabular}

1. Men having sex with men (MSM)

2. Intravenous drug use (IDU) 


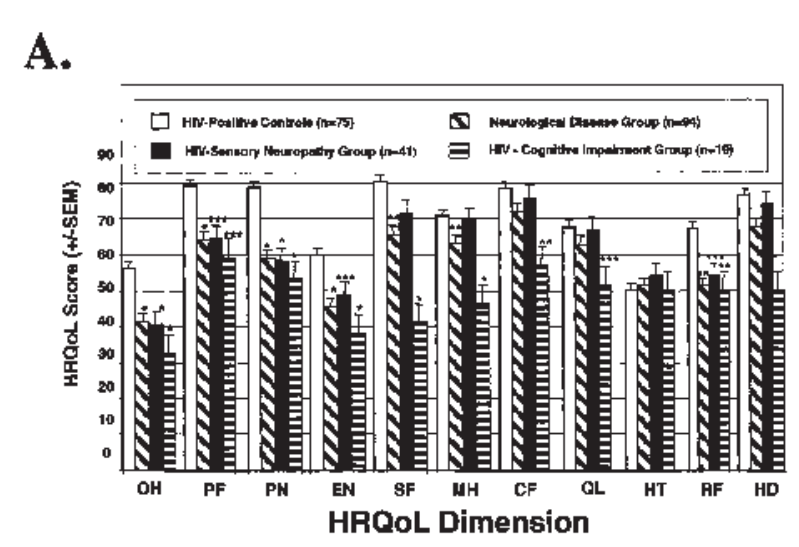

B.

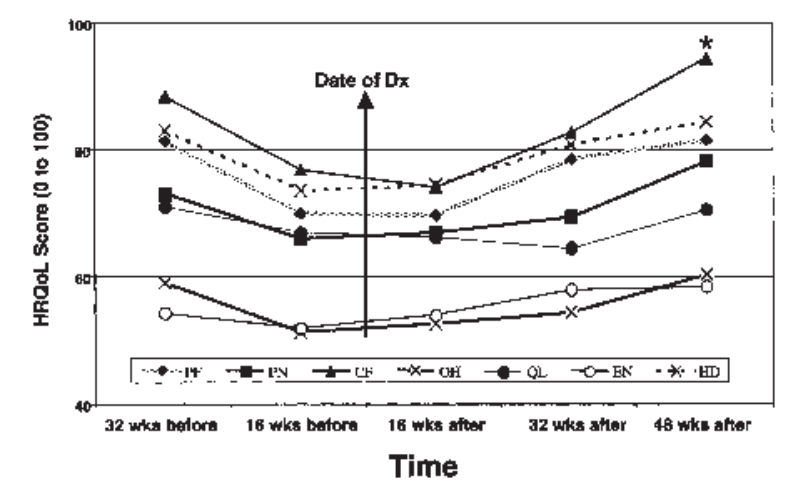

Figure: Health-related quality of life dimensions among HIV/AIDS patients with and without neurological disease. (A) All patients with a neurological disease (NDG) demonstrated lower HRQoL compared to neurological-disease free Controls at baseline interview. Similarly, patients with HIV-related sensory neuropathy and HIV related cognitive impairment showed lower HRQoL scores than Controls in most dimensions. (B) Health-related quality of life subscale dimensions of overall health, pain, physical function, cognitive function, energy level, health distress, and quality of life showed improvement over the 96-week period following the diagnosis of neurological disease. Mental health, social functioning, role functioning, and health transition parameters were unchanged over the same period. $(O H=$ general overall perception of health, $Q L=$ quality of life, $M H=$ mental health, $D \chi=$ diagnosis, $P F=$ physical functioning, $P N=$ level of pain, $E N=$ energy level, $S F=$ social functioning, $R F=$ role functioning, $H D=$ health distress, $H T=$ health transition, $C F=$ cognitive functioning. (ANOVA with post hoc Bonferroni comparisons; ${ }^{*} p<0.05, *^{*} p<0.01$, $* * * p<0.001)$.

$(\mathrm{p}<0.05)$, except for the mental health, cognitive function, and health distress dimensions. Patients with cognitive impairment (HIV-associated dementia, $\mathrm{n}=9$; minor cognitive motor disorder, $\mathrm{n}=10$ ) consistently demonstrated the lowest HRQoL scores for all dimensions examined herein $(\mathrm{p}<0.01)$ except for the health transition dimension. Comparisons among the different neurological groups revealed that the HIV-related cognitive impairment group showed significantly reduced social functioning, mental health, cognitive function and health distress compared to the NDG and the HIV-sensory neuropathy group (Figure). To exclude any bias by selection of patients based on age, sex, education, CD4 level and duration of disease, we compared the NDG with all patients without neurological disease who had received the Medical Outcomes Short-form Health Survey-HIV questionnaire $(n=200)$ in the parent study, revealing that patients with neurological disease scored significantly worse than controls, even when stratified by CD4 level (data not shown). Patients having multiple neurological diagnoses showed lower HRQoL scores in the dimensions of quality of life and social functioning compared to those with a single neurological diagnosis $(\mathrm{p}<0.05)$. Thus, these studies indicated that the HRQoL scores among HIV/AIDS patients with neurological disease were substantially lower than those from patients without neurological disease.

Although the above cross-sectional studies suggested that HRQoL was reduced among HIV/AIDS patients with neurological disease, we wanted to investigate the longitudinal aspects of HRQoL in these patient groups. Prospective analysis of HRQoL dimensions (Figure B) within the NDG group $(n=36)$ revealed a trend of diminishing HRQoL scores (from 32 to 16 weeks) prior to the date of neurological diagnosis and implementation of appropriate treatments followed by a progressive improvement in HRQoL, beginning approximately 16 weeks after the date of neurological diagnosis until 32 weeks after the neurological diagnosis was made. Specific treatments included analgesics and antiretroviral drugs including nucleoside analogues with efficient brain penetration. This trend was evident for the dimensions of physical functioning, pain, cognitive function, overall health, energy, quality of life, and health distress while only the cognitive function dimension demonstrated a significant improvement in HRQoL scores at 48 weeks after the diagnosis of a neurological condition $(\mathrm{p}<0.05)$. The Control group showed no consistent changes over the 80week period for all dimensions, indicating that HRQoL scores are sensitive to clinical intervention in patients with neurological diseases.

\section{Discussion}

The present study reports the first analysis of HRQoL among HIV/AIDS patients targeting those with a range of neurological syndromes. Importantly our study was performed in a community clinic and thus, our population of interest provides a broad and representative perspective of neurological disease among HIV/AIDS patients. We demonstrate that HIV/AIDS patients with neurological syndromes have significantly lower HRQoL scores in compared to age-, sex-, disease durationmatched controls, even after controlling for the level of immunosuppression and treatment. In addition, patients with cognitive impairment consistently demonstrated the lowest HRQoL scores. The prospective analysis revealed a decline in several HRQoL dimensions prior to a neurological diagnosis and treatment implementation. This decline was followed by a trend of progressively improving HRQoL scores in these important dimensions at 16 weeks after the date of the neurological diagnosis and the initiation of specific treatments. Thus, these observations underscore the significant adverse, albeit treatable, impact of neurological disease on HRQoL in patients with HIV/AIDS. 
Health-related quality of life in neurological disease has been shown to be markedly diminished for several neurological diseases including multiple sclerosis, Parkinson's disease and Alzheimer's disease. ${ }^{9}$ The factors contributing to diminished HRQoL scores in these groups of patients include insight into worsened health prognosis, increasing physical disability, and pain that impact negatively on HRQoL. We concentrated on patients with relatively mild cognitive impairment including patients with minor cognitive motor disorder and HIV-associated dementia who continued to live independently. In addition to cognitive impairment requiring attention in the current study, the concomitant presence of depression is another factor that may influence HRQoL although the frequency of hospital admission for a major depressive episode and use of antidepressant drugs did not differ between the NDG and Control groups (data not shown). The effect of HAART on quality of life has been assessed in multiple studies, indicating that the use of HAART improves HRQoL. ${ }^{10}$ In the present study, similar numbers of patients were receiving HAART; for the majority of Control and NDG patients, HAART regimens were comprised of two nucleotide analogues and a protease inhibitor and individual HAART regimens were not likely to affect HRQoL scores. However, 26 patients of those with HIV-sensory neuropathy $(n=41)$ in the present study had distal sensory polyneuropathy caused by HIV infection while the remaining HIV-sensory neuropathy patients had antiretroviral toxic neuropathy. Hence, among those patients with antiretroviral toxic neuropathy, HAART may have had an adverse influence on HRQoL scores. Nonetheless, HRQoL scores did not differ significantly between patients with distal sensory polyneuropathy and antiretroviral toxic neuropathy (data not shown). While the improvement in several HRQoL dimensions following diagnostic and therapeutic interventions within the NDG group was of particular interest, this group of patients represented only a subset of the total group of the NDG. The improvement in HRQoL scores is likely due to an alleviation in symptoms due to assurance of the patients of the diagnosis as well as the effects of specific therapies, as suggested by earlier reports including neurocognitive impairment with specific interventions. ${ }^{11}$ Indeed, our findings underscore the importance of addressing HRQoL concerns among HIV/AIDS patients with neurological disease, especially as intervention appears to improve patients' sense of well-being and may be important considerations in the allocation of health care resources for HIV/AIDS patients.

\section{ACKNOWLEDGEMENTS}

The authors thank Belinda Ibrahim and Andrea Sullivan for assistance with manuscript preparation.

\section{REFERENCES}

1. Sacktor NC, Skolasky RL, Lyles RH, et al. Improvement in HIVassociated motor slowing after antiretroviral therapy including protease inhibitors. J Neurovirol 2000; 6: 84-88.

2. Power C, Gill MJ, Johnson RT. The neuropathogenesis of HIV infection: host-virus interaction and the impact of therapy. Can J Neurol Sci 2002; 29: 19-32.

3. Wu AW. Quality-of-life assessment in clinical research: application in diverse populations. Med Care 2000; 38(II): 130-135.

4. Osowiecki DM, Cohen RA, Morrow KM, et al. Neurocognitive and psychological contributions to quality of life in HIV-1-infected women. AIDS 2000; 14: 1327-1332.

5. Benedict RH, Mezhir JJ, Walsh K, et al. Impact of human immunodeficiency virus type-1-associated cognitive dysfunction on activities of daily living and quality of life. Arch Clin Neuropsychol 2000; 15: 535-544.

6. Krentz HB, Auld MC, Gill MJ. The changing direct costs of medical care for patients with HIV/AIDS, 1995-2001. Can Med Assoc J 2003; 169: 106-110.

7. Janssen R. Nomenclature and research case definitions for neurologic manifestations of human immunodeficiency virustype 1 (HIV-1) infection. Report of a Working Group of the American Academy of Neurology AIDS Task Force [see comments]. Neurology 1991; 41: 778-785.

8. Bouwman FH, Skolasky RL, Hes D, et al. Variable progression of HIV-associated dementia. Neurology 1998; 50: 1814-1820.

9. Novella JL, Jochum C, Jolly D, et al. Agreement between patients' and proxies' reports of quality of life in Alzheimer's disease. Qual Life Res 2001; 10: 443-452.

10. Saunders DS, Burgoyne RW. Evaluating health-related wellbeing outcomes among outpatient adults with human immunodeficiency virus infection in the HAART era. Int J STD AIDS 2002; 13: 683-690.

11. Brew BJ. Evidence for a change in AIDS dementia complex in the era of highly active antiretroviral therapy and the possibility of new forms of AIDS dementia complex. AIDS 2004; 18 (Suppl 1): S75-S78. 